\title{
METODOLOGÍA DE REDUCCIÓN DE SOMBRA DE FALLA: APLICADA EN UNA ZONA DE LA CUENCA LLANOS - DEPARTAMENTO DE CASANARE, COLOMBIA
}

\author{
Claudia Milena Torres-Vega' ${ }^{1}$ Ovidio Amado Almanza-Montero² ${ }^{2}$ Luis Alfredo Montes-Vides ${ }^{\text {* }}$ \\ DOI: http://dx.doi.org/10.18273/revbol.v39n2-2017004 @c) (i) \\ Forma de citar: Torres-Vega, C.M, Almanza-Montero, O.A., y Montes-Vides, L.A. 2017. Metodología de \\ reducción de sombra de falla: Aplicada en una zona de la Cuenca Llanos - Departamento de Casanare, Colombia. \\ Boletín de Geología, 39(2): 57-66.
}

\section{RESUMEN}

La sombra de falla distorsiona la imagen sísmica del bloque yacente de una falla normal o inversa, lo cual se debe a fuertes cambios de velocidad lateral que desvían la trayectoria de los rayos. En la Cuenca Llanos este efecto crea en las imágenes sísmicas falsas estructuras de anticlinales ("pull-up") y sinclinales ("push down o sag"). En este artículo se estudian los factores que generan este efecto y su impacto en las imágenes sísmicas mediante el modelado numérico. Se define una metodología para identificar la sombra y los factores que la causan, y crear el modelo apropiado para la migración PSDM. La metodología se probó en sismogramas sintéticos y se aplicó a una línea sísmica de un sector del Departamento de Casanare-Colombia. Como resultado se obtuvo una imagen en profundidad confiable libre de sombra de falla. Aunque la metodología se usó en un sector con falla normal ésta es aplicable a zonas con fallas inversas.

Palabras clave: Sombra de falla, falsa estructura, modelado numérico, cuenca llanos, Casanare..

\section{METHODOLOGY TO REDUCE FAULT SHADOW: APPLIED IN A SECTOR OF THE LLANOS BASIN - COLOMBIA}

\begin{abstract}
The shadow fault distorts the image of the hanging block of normal and inverse faults, caused by strong lateral velocity changes that deviates the ray path of waves. In the Llanos basin, this effect creates in the seismic images false structures like anticlines (pull up) and synclines (push down or sag). In this paper, the factors that generate this effect and its impact on the seismic images are studied through numerical modeling. It is defined a methodology to identify the fault shadow and its causing factors, and to create the appropriate model for the pre stack depth migration - PSDM. The methodology was tested with synthetic seismograms and applied to a seismic line recorded in a sector of the Departamento de Casanare-Colombia. As a result, a reliable image in depth free of fault shadow was obtained. Even the methodology was used in a sector with normal fault it is applicable to zones with inverse faults.
\end{abstract}

Keywords: fault shadow, false structure, numeric modeling, llanos basin, Casanare.

\footnotetext{
'Departamento de Geociencias, Universidad Nacional de Colombia. Bogotá, Cundinamarca, Colombia. cmtorresv@unal.edu.co, (*)lamontesv@unal.edu.co

${ }^{2}$ Departamento de Física, Universidad Nacional de Colombia. Bogotá, Cundinamarca, Colombia. oaalmanzam@unal.edu.co
} 


\section{INTRODUCCIÓN}

La sombra de falla causa distorsión en la imagen sísmica del bloque yacente de una falla normal o inversa, debido a que los rayos se curvan fuertemente cuando atraviesan la zona fallada lo que modifica los tiempos de tránsito y cambia el carácter hiperbólico de las curvas de corrección dinámica NMO ("Normal Move Out"). Bajo la sombra de falla, la imagen distorsionada muestra complejas estructuras artificiales similares a fallas, chimeneas de gas, plegamientos o potenciales prospectos. Esta incertidumbre incrementa el riesgo de una errónea interpretación estructural y estratigráfica de la sección en tiempo. Para eliminar el efecto se aconseja migrar en profundidad, preferiblemente con el algoritmo Kirchhoff - PSDM ("Pre-Stack Depth Migration"), ya que puede manejar los fuertes cambios de velocidad lateral en la falla (Fagin, 1998; Gochioco et al., 2002). A su vez, la PSDM requiere reflexiones fuertes y un detallado modelo de velocidad lo cual significa que el objetivo de la PSDM sería validar el modelo propuesto. Lo anterior, hace incierto el procesamiento sísmico en la zona bajo la falla ya que no se sabe de antemano si esas estructuras son reales. A fin de identificar la sombra de falla y sus causas se sugiere migrar con la PSDM registros sintéticos no apilados generados en modelos geológicos con fallas para comprobar el correcto posicionamiento de los reflectores deformados en las secciones migradas (Fagin, 1996; Trinchero, 2000). Por otro lado, se han identificado factores que afectan la imagen de la estructura real como el salto y el ángulo de inclinación de la falla (Martínez y Agudelo, 2009) así como los rápidos cambios de velocidad propias de la zona fallada (Birdus and Artyomov, 2010). En el modelo definitivo se propagan ondas en las estructuras geológicas bajo la falla para conocer donde se reflejan (Alaei, 2012), se actualiza el modelo de velocidad con los registros de pozo y se correlaciona con las estructuras (Bain, 2015). Una tomografía restringida a la zona de falla permitiría construir un modelo de velocidad en profundidad de alta resolución para la PSDM (Birdus, 2007) y la curva de conversión tiempo-profundidad del modelo (Chermak et al., 2009).

En la cuenca Llanos la secuencia sedimentaria MesoCenozoica es frecuentemente cortada por fallas por lo que esclarecer estas anomalías disminuiría el riesgo de proyectos de exploración y producción de prospectos en tales zonas. El área de estudio se ubica en el municipio de Maní a $70 \mathrm{Km}$ de Yopal capital de Departamento del Casanare - Cuenca Llanos, entre las coordenadas geográficas mostradas en la FIGURA 1A. Se contó con un "check-shot", registros sónicos y de densidad de los pozos E-1, E-2 y E3 ubicados paralelamente a las líneas sísmicas 2D: R-2005-01, R-2005-02 y R-2005-03. De estas líneas, la R-2005-03 es la más cercana al pozo E-1. El recuadro punteado en la sección apilada de la línea R-2005-03 de la FIGURA 1B muestra un levantamiento $\mathrm{y}$ un hundimiento de los reflectores al tope y base de la Fm. Carbonera.
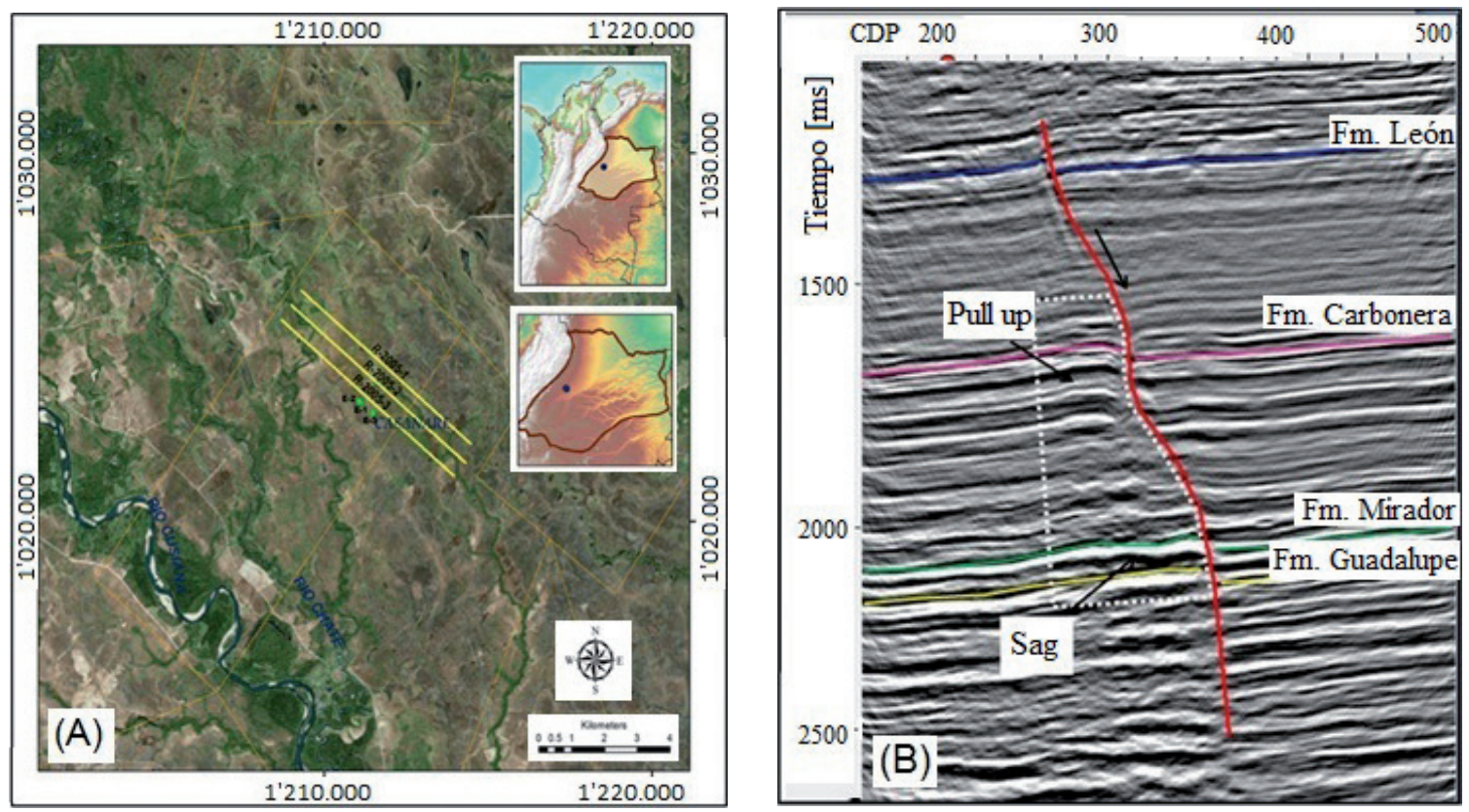

FIGURA 1. A. El mapa muestra la Cuenca Llanos - Colombia y la ubicación de las líneas 2D R-2005-01, R-2005-02 y R-200503, y los pozos E-1, E-2 y E3. B. En la línea sísmica R-2005-03, la sombra de la falla distorsiona la imagen en el bloque yacente desde el CDP 240 al 360 en las formaciones León, Carbonera, Mirador y Guadalupe. 


\section{GEOLOGÍA}

La Cuenca Llanos es un monoclinal suave de rumbo NE-SW interpretado al SE como un anticlinal truncado contra la falla, donde los prospectos se asocian al tren de fallas normales con importantes estructuras productoras. La historia geológica comienza en el Paleozoico con una fase extensional ("rifting") que permitió la acumulación de sedimentos siliciclásticos sobre un basamento cristalino precámbrico. En el Jurásico - Cretácico Tardío la cuenca fue el brazo oriental de un gran sistema extensional cubierto por depósitos de plataforma marina y a partir del Maestrichtiano se convirtió en una cuenca de antepaís. Durante el Triásico - Cretáceo Inferior la cuenca era tipo extensional de retro-arco, mientras en el Cretáceo Superior pasó a ser pasiva de retroarco. La cuenca actuó como una de tipo plataforma en el Paleoceno Maestrichtiano y evolucionó a una de ante-país en el Mioceno. Durante la transición del Cretácico al Paleoceno la principal deformación dio origen a la acreción de la cordillera Occidental, lo cual cambió los ambientes de depositación de la cordillera Oriental y la cuenca de los llanos. Esto explica el aporte de depósitos marinos en la cuenca y algunos aportes fluviales provenientes de la parte oeste de la cuenca (Cooper et al., 1995). Durante el Eoceno - Oligoceno se reactivaron fallas en el Valle Inferior del Magdalena las cuales no causaron deformaciones importantes en la cuenca Llanos. Luego, en el Oligoceno y Mioceno, el continuo levantamiento de la cordillera Oriental generó un espacio de acomodación en la parte distal de la cuenca, la reactivación de fallas y la migración hacia el este del alto flexural (Bayona et al., 2008). El pulso de deformación de la cordillera Oriental más fuerte y de mayor impacto en la cuenca Llanos ocurrió durante el Mioceno Medio - Tardío, la cual condicionó la depositación aluvial a una fluvial registrada en la formación Guayabo (Bayona et al., 2008), que contiene depósitos erosionados de la cordillera Oriental. La deformación y crecimiento de la cordillera Oriental aún sigue activa lo que causa periódicos movimientos telúricos en la parte oeste de la cuenca y algunos movimientos en su parte central.

La FIGURA 2 muestra la columna estratigráfica generalizada de la cuenca Llanos que contiene una sección Paleozoica, unidades Cretácicas (Ubaque, Gachetá y Guadalupe) y Cenozoicas (Barco, Los Cuervos, Mirador, Carbonera, León, Guayabo, Necesidad y Cometa).

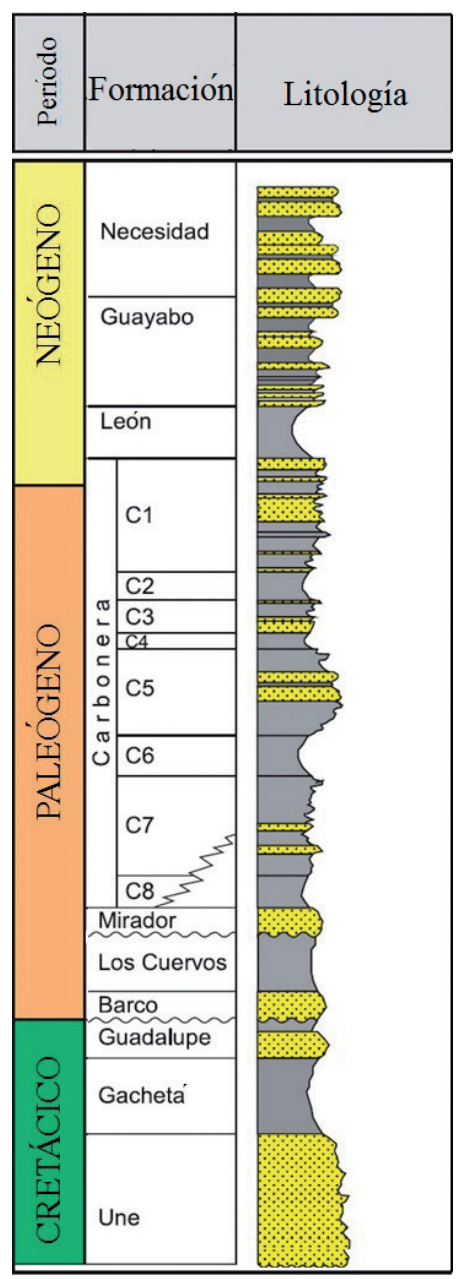

FIGURA 2. Columna estratigráfica generalizada de la Cuenca Llanos con las secciones Paleozoicas, Mesozoicas y Cenozoicas presentes en el sector estudiado.

La unidad Paleozoica consiste en su base de cuarcitas, conglomerados, calizas, grauvacas con ligero metamorfismo e interposiciones de lodolitas y arcillolitas. La parte superior está constituida por lutitas fosilíferas grises a negras predominantes sobre arenitas y limolitas de edad Ordovícico. El espesor y disposición de esta unidad sugiere un depósito de plataforma somera, donde la sedimentación marina es preponderante sobre depósitos litorales de granulometría más gruesa. La Formación Ubaque - con un espesor promedio de $90 \mathrm{~m}$ - se presenta discordante sobre el Paleozoico y consiste de cuarzoarenitas macizas de grano fino a medio, localmente conglomeráticas, inter-estratificadas con capas delgadas de limolitas carbonosas y arcillolitas. La Formación Gachetá es una sucesión de lutitas grises y negras e inter-estratificaciones locales de capas 
delgadas de calizas y arenitas fino-granulares. En el sector de estudio, presenta un espesor promedio de 110 m. La Formación Guadalupe es una sucesión de arenitas cuarzosas de grano fino a medio, con delgadas intercalaciones de lodolitas, porcelanitas y localmente carbón. El espesor en el sector de este estudio fluctúa entre 50 y $90 \mathrm{~m}$. Una caída grande en el nivel relativo del mar en el Paleoceno condicionó un cambio en el ambiente de depósito, lo cual causó un hiato estratigráfico importante en la cuenca Llanos. Por lo tanto, no se registra la presencia de las formaciones Barco y Los Cuervos en el sector bajo estudio, caracterizado por una inconformidad de carácter regional entre la suprayacente Formación Mirador y la subyacente Formación Guadalupe. La Formación Mirador está constituida por areniscas de grano grueso a muy fino, en algunos casos con cemento calcáreo, limolitas grises, pardas microcarbonosas y lutitas grises verdosas. Las areniscas son de mayor grosor hacia la base, de colores blancos, grises, a veces verdosas, cuarzosas, eventualmente calcáreas y en algunos casos con chert y glauconita. En la cuenca se ha documentado un espesor de 3000 a 3500 metros de depósitos clásticos de origen continental y depositados en los últimos 10 millones de años como respuesta al levantamiento y erosión de la Cordillera Oriental. Estos depósitos, que se identifican en el área como pertenecientes a las formaciones Guayabo, Necesidad y Cometa, presentan en dicha área espesores superiores a 1200 metros.

En el sector estudiado la Formacion León es una unidad de baja velocidad considerada una roca sello regional de aproximadamente $370 \mathrm{~m}$ de espesor constituida por arcillolitas y lodolitas de carácter marino somero a lacustre. La Formación Carbonera con espesor aproximado de $600 \mathrm{~m}$ corresponde a un ambiente continental transicional, y consta de 8 unidades de las cuales el nivel arenoso $\mathrm{C} 1$ es el producto de depósitos de canales fluviales. La Formación Mirador, de $23 \mathrm{~m}$ de espesor, tiene desarrollos arenosos que corresponden a depósitos de canales, de bahías y de estuarios. La Formación Guadalupe, con un espesor aproximado de $11 \mathrm{~m}$, está constituida predominantemente por areniscas y algunas intercalaciones de limolitas y lodolitas. Al profundizarse en dirección SE-NO, la cuenca Llanos (extensional y de ante-país) contiene trampas estructurales asociadas a una falla normal antitética de alto ángulo con desplazamiento vertical variable de hasta $190 \mathrm{~m}$. Los mayores saltos se observan al tope de las formaciones Cenozoicas León y Carbonera, y los menores en las Cretácicas Gachetá y Ubaque. El trazo de la falla es claro en las formaciones Guayabo y León, pero no en las formaciones Carbonera y Mirador debido a los cambios laterales de velocidad, tanto que pareciera existir más de una falla en la imagen, como se ve en la FIGURA 1B. Las secciones sísmicas de algunas áreas evidencian distorsiones en el bloque yacente bajo el plano de falla, como estructuras anti-formes asimétricas ("roll over") o como variaciones de buzamiento en los reflectores, lo que impide la definición de prospectos y/o el desarrollo de campos. Por su carácter extensional es poco probable la presencia de estructuras de tipo anticlinal. Estructuras interpretadas como anticlinales que resultaron con pozos secos son posiblemente "pull ups" causados por la falla.

\section{PROCEDIMIENTO}

Para estudiar el efecto de la zona de falla en regímenes distensivo y/o compresivo se construyeron dos modelos de capas planas horizontales y paralelas. El primero, afectado por una falla normal y el segundo, por una falla inversa. Se simuló la propagación de ondas en estos modelos, se generaron los sismogramas sintéticos y se procesaron hasta obtener sus secciones apiladas. En los pozos E-1, E-2 y E-3 se identificaron los topes de las formaciones Guayabo, León, Carbonera, Mirador y Guadalupe, los cuales fueron estimados en profundidad mediante una curva tiempo-profundidad y amarrados a la sección migrada en tiempo de la línea R-2005-03 cuya interpretación suministró el modelo inicial del subsuelo. Con el modelo en tiempo, que incluye la estructura y los estratos, se construyó el prototipo de modelo velocidad/ densidad en profundidad de acuerdo a los registros del pozo E-1, con una falla normal que atraviesa tres unidades. Se crearon 16 modelos que combinan saltos de falla de $60,90,150$ y $300 \mathrm{~m}$ con ángulos de falla de $15^{\circ}, 30^{\circ}, 45^{\circ}$ y $60^{\circ}$. Se simularon adquisiciones sísmicas para cada modelo con un diseño similar al usado en el registro de la línea R-2005-03. Los registros sintéticos generados se procesaron inicialmente en tiempo y luego en profundidad con la misma secuencia aplicada para obtener la sección PSTM de la línea R-2005-03. El procesamiento de los registros sísmicos se hizo con el software ProMAX 2D, mientras que la creación de modelos en profundidad y la consecuente propagación de la onda en el modelo, para generar los sismogramas sintéticos correspondientes a las geometrías de adquisición, se hicieron con la herramienta Tesseral-Pro 2D. Se estudiaron los efectos de salto y ángulo de falla en las 16 imágenes obtenidas, y se compararon con la imagen PSTM de la línea R-2005-03 para identificar efectos similares en esta última. El modelo cuya imagen respondía a los efectos observados en sección PSTM de la línea se seleccionó como insumo inicial de la PSDM aplicada a los registros CDP de la línea. El modelo fue 
actualizado durante las iteraciones de la migración y sus valores de velocidad restringidos por los registros de pozo.

\section{MODELADO NUMÉRICO}

La variación lateral de velocidad y la presencia de unidades de baja velocidad causan anomalías en los tiempos de viaje, lo que produce plegamientos y reflexiones discontinuas interpretables como fallas satélites. Las deformaciones en los reflectores se deben a los tiempos de viaje de los rayos mientras las discontinuidades las causan las deflexiones de los rayos en la zona fallada. La FIGURA 3A contiene el modelo de capas planas paralelas cortadas por una falla normal en un régimen extensional, donde la velocidad aumenta en profundidad pero incluye una capa de baja velocidad. La FIGURA 3B muestra la correspondiente sección apilada, donde, bajo la falla, los reflectores entre 0,05 y 0,10 segundos se ven plegados y los reflectores entre 0,10 y 0,20 segundos muestran un sinclinal y un anticlinal. La imagen apilada induciría una interpretación diferente al modelo con reflectores planos bajo la falla. La FIGURA 4A muestra el modelo bajo un régimen compresivo con una falla inversa donde el bloque colgante cabalga sobre el yacente. La FIGURA 4B contiene la correspondiente sección apilada obtenida al procesar los registros sintéticos generados en el modelo. Bajo la falla, en los reflectores entre 0,0 y 0,10 segundos se ven pequeños pliegues cerca al plano de falla mientras los reflectores entre 0,20 y 0,25 segundos muestran anticlinales y anticlinales espurios. El efecto de sombra en es más pronunciado que en el extensional debido al fuerte contraste de velocidad, la mayor deformación y desplazamiento del bloque cabalgante.
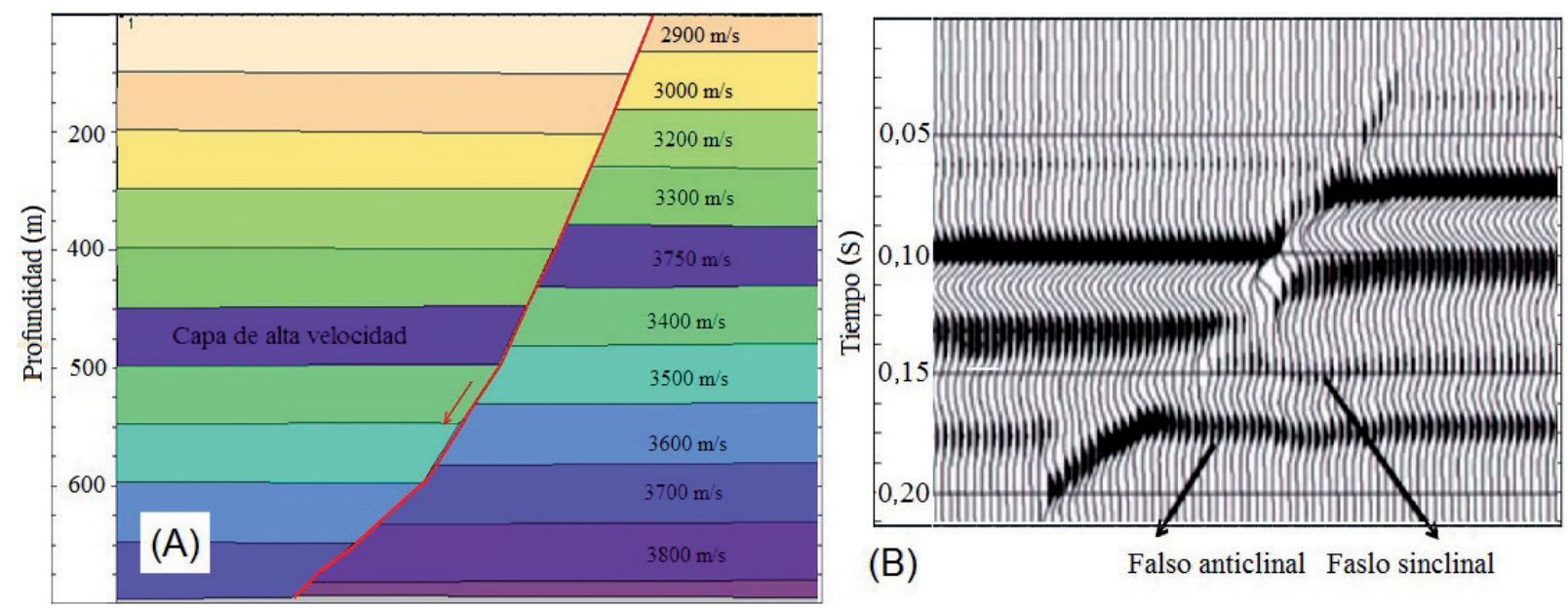

FIGURA 3. A. Modelo de esfuerzo distensivo con falla normal e inversión de velocidad y B. La respuesta sísmica "zero offset" del modelo con levantamientos y hundimientos de los reflectores bajo la falla.
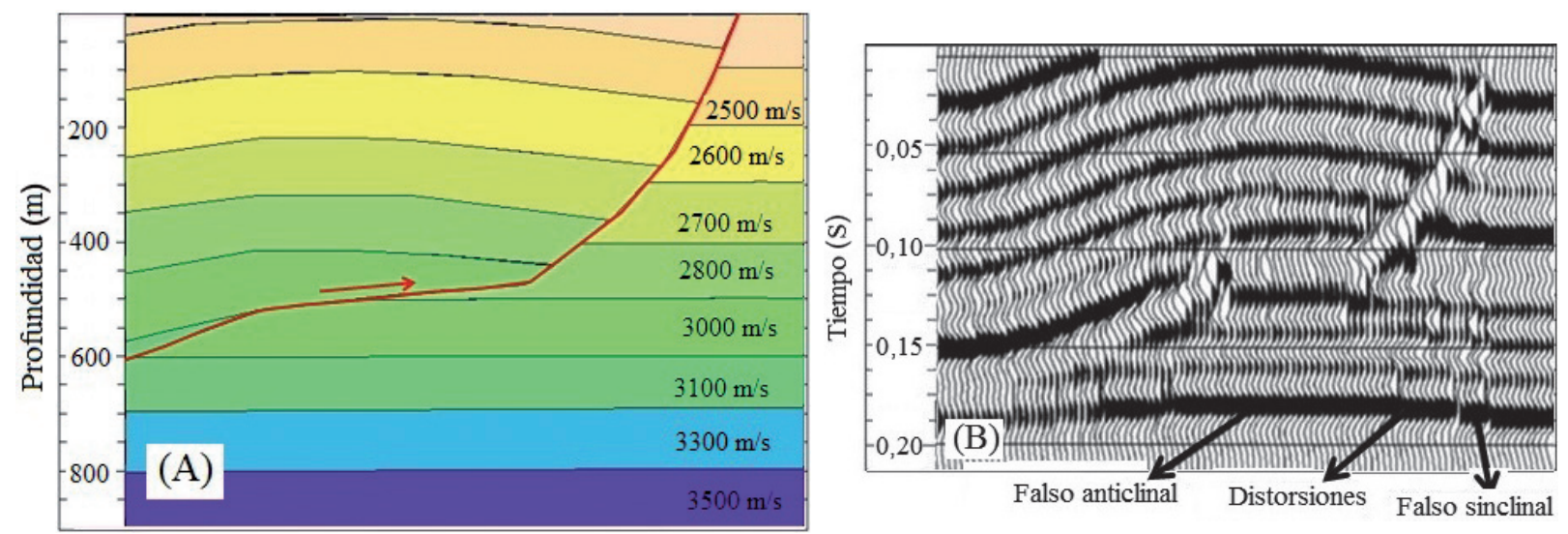

FIGURA 4. A. El modelo de capas planas horizontales y paralelas con una falla inversa bajo un régimen compresivo y B. sección apilada correspondiente al modelo con reflectores fuertemente distorsionados en la zona de sombra de falla. 
La formación León crea una inversión de velocidad al yacer entre dos formaciones de mayor velocidad, por lo que se estudió la respuesta sísmica en modelos con y sin inversión de velocidad. La FIGURA 5A muestra el modelo de una capa de alta velocidad entre dos capas de baja velocidad con una falla normal inclinada y rayos "zero-offset" que recorren el modelo desde la superficie hasta la base de la capa de alta velocidad. En una capa de alta velocidad el tiempo de viaje del rayo (medido por su longitud) es menor que en una de menor velocidad. Así, el rayo 1 recorre solo la capa de baja velocidad mientras los otros viajan parcialmente por la de alta velocidad, por lo que sus tiempos de viaje serán menores cuando el recorrido sea mayor en esa capa y menor en la de baja velocidad. Los tiempos de los rayos 2, 3 y 4 disminuyen al viajar por la capa de alta velocidad por lo que la anomalía en tiempo bajo la falla levanta el reflector en la base de la capa de alta velocidad ("pull-up"). Por el contrario, la FIGURA 5B muestra un modelo fallado en tiempo con una capa de baja velocidad entre dos de alta velocidad (inversión de velocidad). Al cruzar la falla, el rayo demora más tiempo al viajar en la capa de baja velocidad que en la de alta velocidad, por lo tanto el tiempo de viaje del rayo 1 es menor que el del rayo 2, tiempo que aumenta para los rayos 3 y 4 . La anomalía en tiempo asociada en este caso hace descender el reflector en la base de la capa de baja velocidad ("sag"). El levantamiento o hundimiento del reflector depende de las velocidades, pero según la ley de Snell, afectan el ángulo de refracción de los rayos al cruzar la falla. En la FIGURA 5A el rayo 1 mantiene su trayectoria recta, pero parte de la energía de los rayos 2, 3 y 4 se refracta hacia la izquierda bajo la falla y parte refleja hacia la derecha. A ángulos de incidencia superior al crítico no cruzará la falla, lo cual oscurece la imagen bajo la falla y debilita la continuidad del reflector. En la FIGURA 5B, el rayo 1 mantendrá su trayectoria recta mientras parte de la energía de los rayos 2, 3 y 4 se refractará hacia la izquierda y parte se reflejará a la derecha. En consecuencia se debilita la continuidad del reflector bajo la falla. La FIGURA $5 \mathrm{C}$ muestra una sección "zero-offset" generada en el modelo de la FIGURA 5D que tiene una falla normal de $45^{\circ}$ de inclinación y $300 \mathrm{~m}$ de salto. Las dos situaciones estudiadas en las FIGURAS 5A y 5B son replicadas en el modelo de la FIGURA 5D: la primera, en la parte superior del modelo donde la Formación León supra-yace la Formación Carbonera y la segunda, en la parte inferior del modelo donde la Fm. León subyace la Fm. Guayabo (mayor velocidad). Alrededor de 0.3 segundos bajo la falla en la FIGURA 5C, el reflector tope de la Fm. León se dobla levemente hacia abajo cerca a la falla. Por otro lado, bajo la falla el reflector tope de la Fm. Carbonera (a 0.6 segundos) se dobla hacia arriba igual a lo mostrado por la FIGURA 5B. Al comparar el reflector tope de la Fm. Mirador a ambos lados de la falla, a 1.9 segundos en la FIGURA 5C el reflector muestra un débil y discontinuo sinclinal consecuencia del doblamiento de los rayos con difracciones en puntos donde los reflectores terminan contra la falla. A la derecha de la falla todos los reflectores se mantienen planos, paralelos y horizontales con difracciones en aquellos puntos donde pinchan la falla.
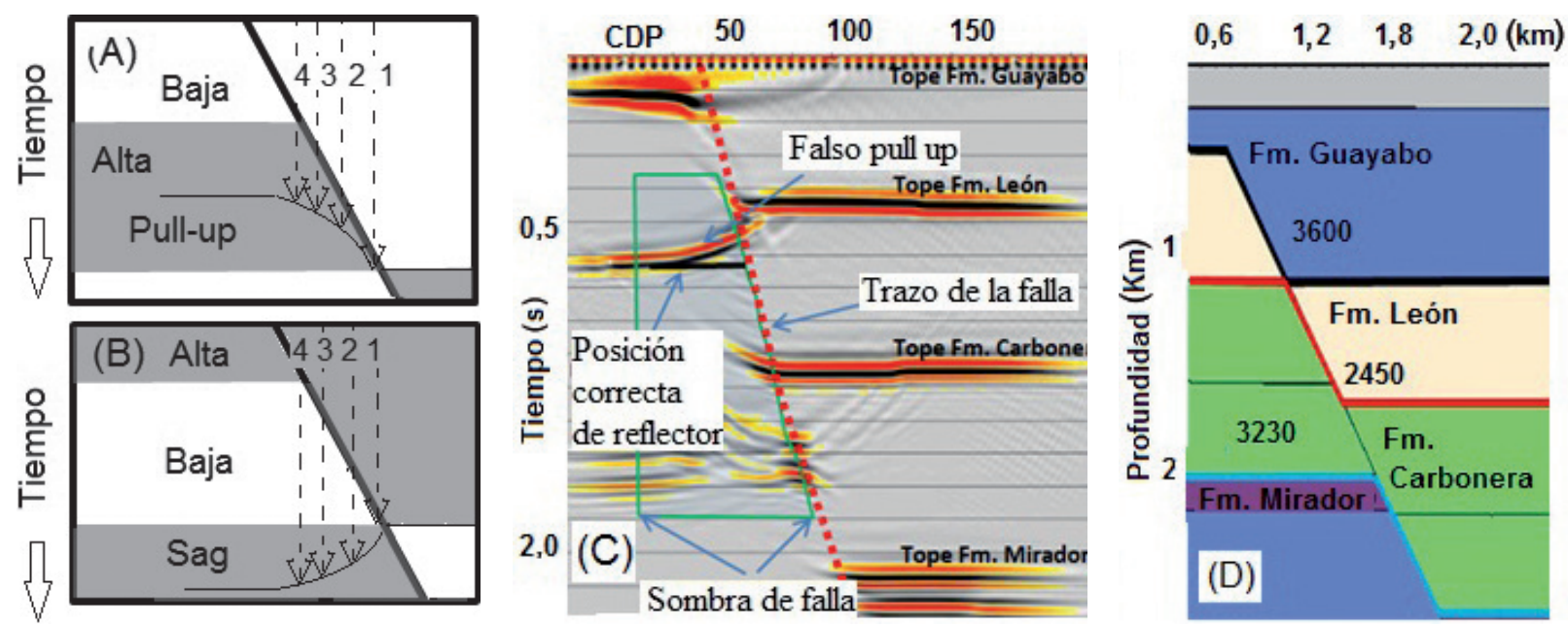

FIGURA 5. A. El modelo con una capa de alta velocidad entre dos capas de baja velocidad muestra levantado el reflector base de la capa de alta velocidad. B. El modelo con una capa de baja velocidad entre dos capas de alta velocidad muestra hundido el reflector base de la capa de baja velocidad. C. La imagen apilada muestra bajo la falla un hundimiento al tope de la Formación León, un levantamiento al tope de la Fm. Carbonera y reflectores difusos y distorsionados de la Fm. Mirador. D. Modelo de capas planas, horizontales y paralelas fallado normalmente con velocidades de las formaciones Guayabo, León, Carbonera y Mirador, del cual se obtuvo la sección apilada en la FIGURA 5C. 
Las 16 adquisiciones sísmicas (con la geometría de la línea R-2005-03) de los 16 modelos (combinan saltos de $60,90,150$ y $300 \mathrm{~m}$ y ángulos de falla de $15^{\circ}, 30^{\circ}, 45^{\circ}$ y $60^{\circ}$ ) generaron registros que se procesaron en tiempo y en profundidad al aplicar la misma secuencia de algoritmos usada para lograr la imagen de la FIGURA 1B. La FIGURA 6A contiene el modelo de velocidades con salto de $60 \mathrm{~m}$ e inclinación de falla de $15^{\circ}$ y la FIGURA 6B la sección apilada obtenida del modelo. Las FIGURAS 6C, 6D y 6E muestran las secciones sísmicas obtenidas de los modelos con ángulo de $15^{\circ}$ y saltos de $90 \mathrm{~m}, 150 \mathrm{~m}$ y $300 \mathrm{~m}$ respectivamente. En la FIGURA 6B el efecto de sombra es casi imperceptible pues los reflectores ligeramente deformados bajo la falla están muy cerca de sus correctas posiciones. Esas deformaciones se hacen más evidentes en la FIGURA 6C al aumentar en el modelo el salto de 60 a $90 \mathrm{~m}$, especialmente al tope de la Fm. Carbonera donde se nota un levantamiento difuso al igual que en el tope de la Fm. Mirador. Cuando el salto de falla alcanza los $150 \mathrm{~m}$, la sombra de falla en la imagen sísmica en la FIGURA 6D muestra un levantamiento claro al tope de la Fm. Carbonera por encima de su posición correcta igual que al tope de la Fm. Mirador que luce levantado y distorsionado. En la FIGURA 6E, al alcanzar el salto de falla $300 \mathrm{~m}$, la distorsión por la sombra es extremo con reflectores difusos y mal posicionados. La secuencia de las FIGURAS 6B, 6C, $6 \mathrm{D}$ y $6 \mathrm{E}$ indica que el efecto por sombra aumenta con el salto. Por otro lado, la FIGURA 7 muestra una secuencia de secciones apiladas en función del ángulo de falla afecta la imagen. La FIGURA 7A muestra el modelo fallado con salto de $60 \mathrm{~m}$ e inclinación de $15^{\circ}$ en la falla, mientras la FIGURA 7B despliega la correspondiente sección sísmica. Las FIGURAS 7C, 7D, 7E y 7F contienen las secciones correspondientes a los modelos con salto de falla de $60 \mathrm{~m}$ y ángulos de $30^{\circ}, 45^{\circ}, 60^{\circ}$ y $75^{\circ}$. Tal secuencia muestra que la sombra es mayor a ángulos menores y que el impacto del ángulo es menor al causado por el salto.
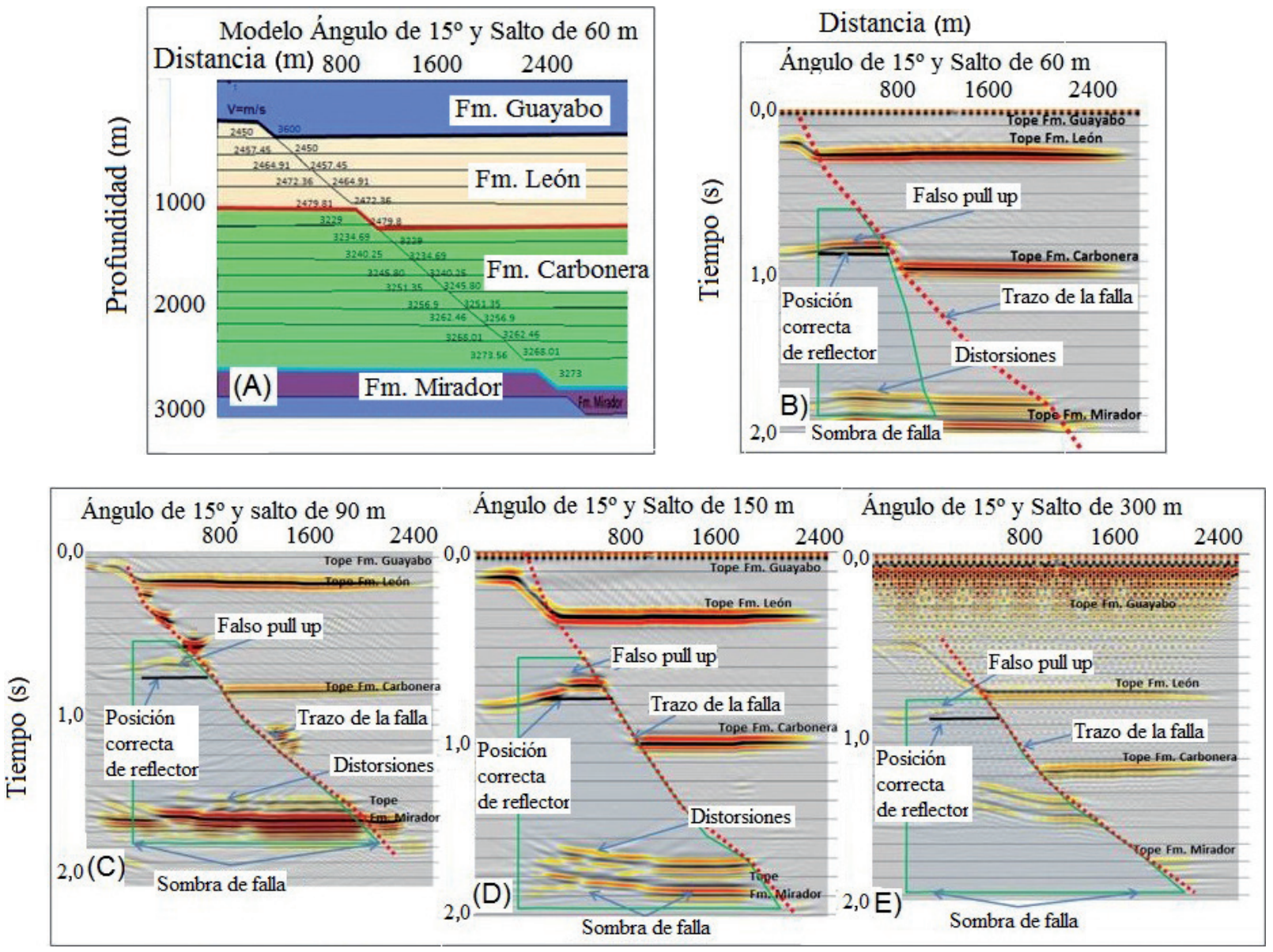

FIGURA 6. A. Modelo de velocidades con salto de $60 \mathrm{~m}$ y $15^{\circ}$ de inclinación de falla, los demás modelos varían solo en el salto de falla. B. Sección sísmica de modelo con salto de $60 \mathrm{~m}$. C. Sección sísmica de modelo con salto de $90 \mathrm{~m}$. D. Sección sísmica de modelo con salto de $150 \mathrm{~m}$. E. Sección sísmica de modelo con salto de $300 \mathrm{~m}$. 

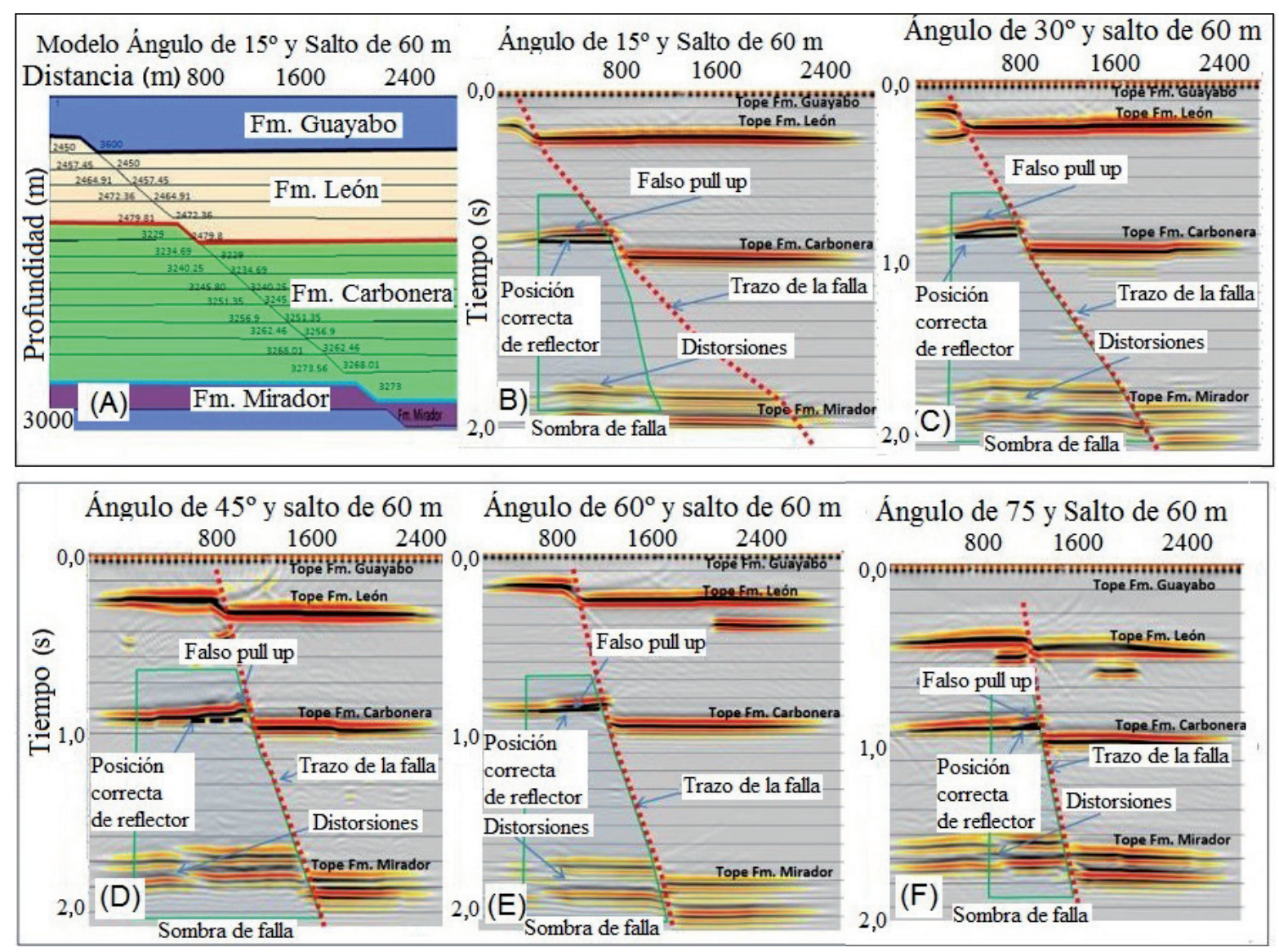

FIGURA 7. A. Modelo de velocidades con salto de $60 \mathrm{~m}$ y $15^{\circ}$ de inclinación de falla, los demás varían solo en el buzamiento de la falla. B. Sección sísmica de modelo con ángulo de $15^{\circ}$. C. Sección sísmica de modelo con ángulo de $30^{\circ}$. D. Sección sísmica de modelo con ángulo de $45^{\circ}$. E. Sección sísmica de modelo con ángulo de $60^{\circ}$. F. Sección sísmica de modelo con ángulo de $75^{\circ}$.

El resultado del proceso antes descrito se resume en una serie de pasos para identificar y atenuar el efecto de la sombra de falla: A) Identificar hundimientos y levantamientos de reflectores bajo la falla. B) Determinar el tipo de falla de acuerdo con el régimen tectónico en la zona (compresivo/extensivo) y estimar el ángulo y el salto de falla. C) Construir un modelo en profundidad con cambios laterales de velocidad en la falla cuyos valores sean consistentes con los de un registro sónico. D) Realizar el modelado numérico para confirmar la sombra de falla con levantamientos o hundimientos de los reflectores en las zonas con variación lateral de velocidad, observados en la imagen sísmica real. E) Usar el modelo escogido como el modelo inicial para la migración PSDM. F) Actualizar el modelo en cada iteración con énfasis en las zonas bajo falla, donde la sección sísmica real exhiba "pull-up" o "sag", hasta obtener la imagen correcta (Quigley et al., 2012). La metodología antes descrita es aplicable a zonas con fallas inversas con la salvedad que las zonas de cabalgamiento se caracterizan por su baja calidad de imagen, consecuencia de las múltiples y curvadas trayectorias de los rayos y la poca iluminación del bloque colgante. Por ende, el éxito de la PSDM depende del modelo de velocidad consistente con registro de pozo que caracterice la litología del sector en consideración.

La FIGURA 8A muestra la sección sísmica con reflectores de la Formación Carbonera con forma anticlinal en el bloque yacente mientras en la base se distinguen distorsiones en la geometría de la estructura bajo la falla, lo cual puede ser causado por la sombra de falla. Se identifica la falla normal con componente de rumbo con salto estimado de $90 \mathrm{~m}$ y máximo ángulo de $45^{\circ}$, en concordancia con la tectónica distensiva del área de la Cuenca Llanos.

Se creó un modelo en profundidad con estas características y se generaron datos sintéticos que fueron luego procesados hasta lograr la sección apilada de la FIGURA 8B. Los reflectores tope y base de la Formación Carbonera muestran igual comportamiento en las FIGURAS 8A y 8B, con un "pull up" al tope el reflector con igual geometría, de lo cual se deduce que el modelo se aproxima la del subsuelo. 

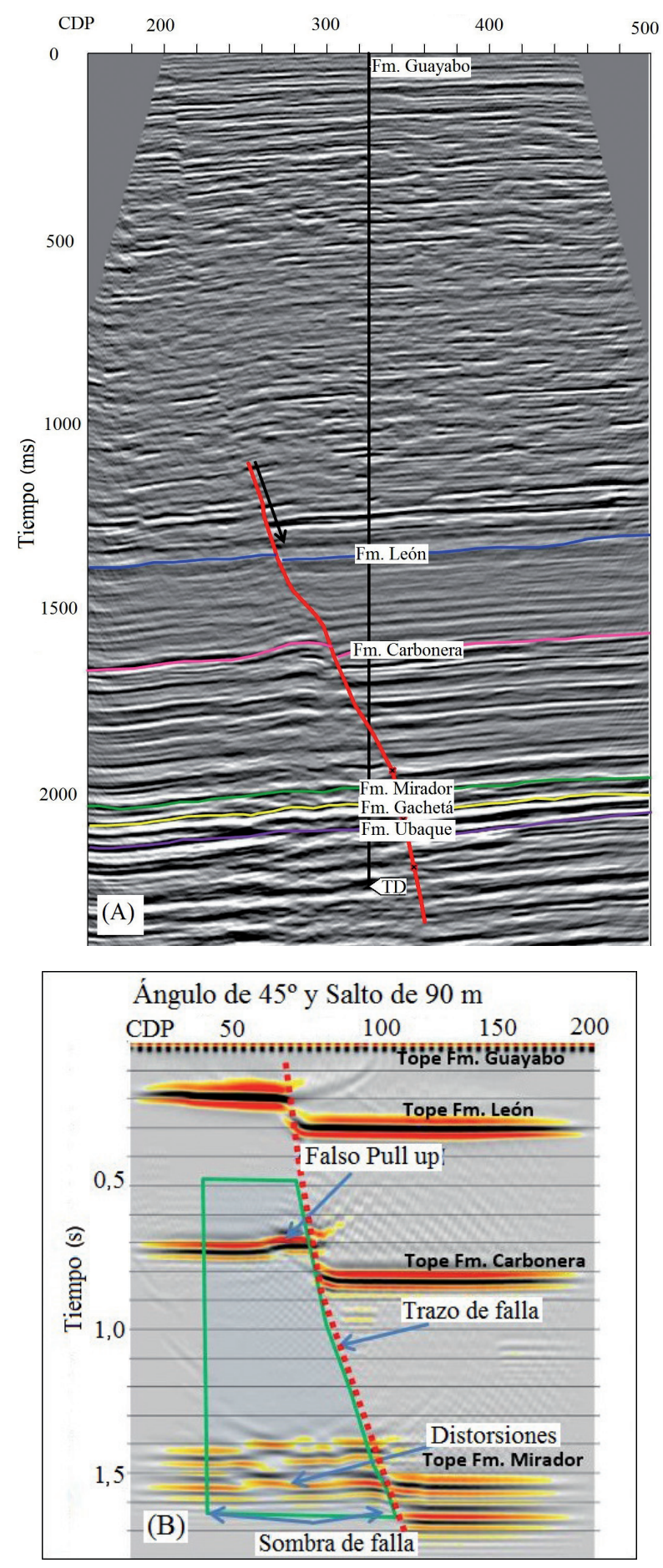

FIGURA 8. A. Interpretación en tiempo de la línea R-200503 donde bajo la falla se observan hundimientos en la Fm. Carbonera y un levantamiento en su tope. B. Sección sísmica obtenida de procesar los registros del modelo con $90 \mathrm{~m}$ de salto e inclinación de falla de $45^{\circ}$. Las dos imágenes se obtuvieron con la misma secuencia de procesamiento.

La FIGURA 9A muestra la imagen PSDM obtenida mediante el procesamiento convencional, donde la sombra de falla causa el levantamiento de los reflectores, fuertes cambios en sus buzamientos y distorsiones en el trazo del plano de la falla. Se construyó el modelo inicial en profundidad con salto de $90 \mathrm{~m}$ y ángulo máximo de $45^{\circ}$, con perfil de velocidad de las formaciones tomadas del registro sónico, el cual fue utilizado junto con los registros CDP sin apilar en la migración en profundidad - PSDM. El registro sónico permitió actualizar en detalle la zona de falla del modelo inicial. El nuevo modelo junto con los registros CDP permitió obtener la sección PSDM que se ve en la FIGURA 9B. Al comparar con la FIGURA 9A, la FIGURA 9B muestra bajo la falla los reflectores mejor definidos y sin estructuras espurias, los cuales presentan cambios laterales en amplitud que facilitan un trazo más definido de la falla.
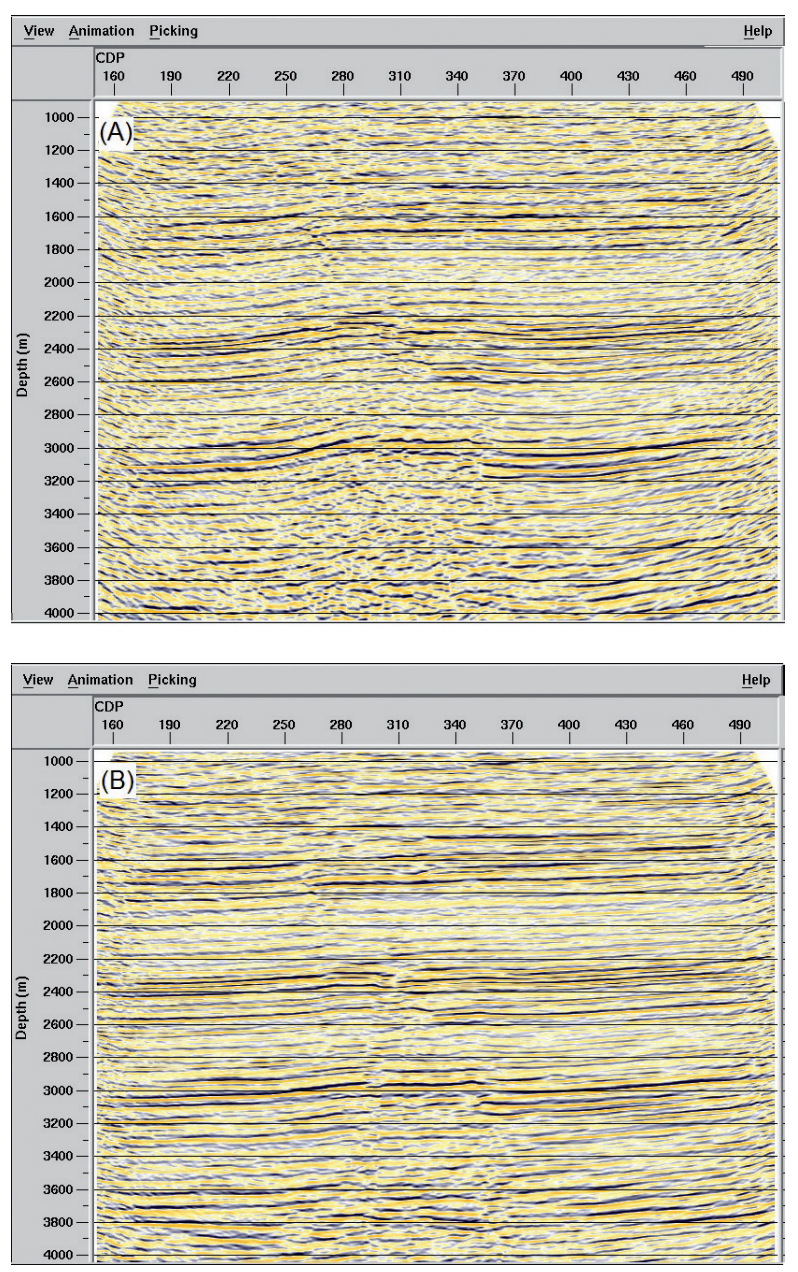

FIGURA 9. A. La imagen migrada en profundidad - PSDM de la línea R-2005-03, obtenida del modelo inicial, muestra falsos anticlinales bajo la falla. B. La imagen migrada en profundidad - PSDM de la línea R-2005-03, obtenida del modelo actualizado con información de inclinación y salto de falla, muestra los reflectores con una geometría acorde con el régimen de esfuerzos de la cuenca Llanos. 


\section{CONCLUSIONES}

Los efectos bajo la sombra causados por el salto y el ángulo de falla se estudian al simular la propagación de ondas en modelos 2D. Mediante modelamiento en zonas con fallas normales e inversas se observa el efecto de sombra causado por el gradiente de velocidad a través de la falla. Como resultado se propone una metodología para atenuar la distorsión por sombra de falla en el bloque colgante, que también es aplicable a zonas con fallas inversas. La metodología se aplicó en un sector de la Cuenca Llanos que tiene registros de pozo y una línea sísmica 2D, cuya interpretación suministró el modelo de velocidad en profundidad en el que la propagación de las ondas replicó las estructuras espurias bajo la falla. El modelo de la PSDM convencional fue modificado en la zona de falla y refinado con un perfil de velocidad ajustado a un registro de pozo. La migración PSDM con el nuevo modelo suministró una imagen que difiere de la anterior imagen PSDM obtenida. Acorde con el régimen distensivo de la cuenca en el sector considerado, la imagen en profundidad suministrada no tiene estructuras falsas bajo la falla y sus reflectores mejor definidos muestran cambios laterales en amplitud que definen mejor el trazo de la falla.

\section{AGRADECIMIENTOS}

Los autores manifiestan agradecimiento a la Universidad Nacional de Colombia y en particular al programa de Maestría en Geofísica. De igual modo extendemos nuestros agradecimientos a la compañía Petróleos Sudamericanos por el acceso a los datos y a las compañías CGG e Inforpetrol por la asesoría y el acceso al software empleado. Finalmente, gracias a los evaluadores cuyas sugerencias contribuyeron a la mayor claridad del documento.

\section{REFERENCIAS}

Alaei, B. 2012. Seismic Modeling of Complex Geological Structures. In: Kanao, M. (Ed.). Seismic Waves - Research and Analysis. InTech, Chapter 11.

Bain, R. 2015. Pitfalls in the seismic interpretation of fault shadow events - Vicksburg formation of south Texas. Interpretation, 3(1): SB17-SB22.

Bayona, G., Cortés, M., Jaramillo, C., Ojeda, G., Ariztizabal J., and Reyes, A. 2008. An integrated analysis of an orogen-sedimentary basin pair: Latest Cretaceous-Cenozoic evolution of the linked Eastern Cordillera orogen and the Llanos foreland basin of
Colombia. Geological Society of America Bulletin, 120(9-10): 1171-1197.

Birdus, S. 2007. Resolving fault shadow problem by Fault Constrained Tomography. ASEG Extended Abstracts: 19th Geophysical Conference. Perth, Western Australia. pp. 1-4.

Birdus, S., and Artyomov, A. 2010. Fault shadow distortions on 3D seismic data and their removal by depth processing. ASEG Extended Abstracts: 21st Geophysical Conference: pp. 1-4.

Chermak, A., Veloza, G., and Ramón, J. 2009. Depth conversion in the Llanos basin: Workflow to reduce fault shadow effect. X Simposio Bolivariano Exploración Petrolera en Cuencas Subandinas, Cartagena, Colombia, pp. 12-16.

Cooper, M., Addison, F., Alvarez, R., Coral, M., Graham, R., Hayward, A., Howe, S., Martinez, J., Naar, J., Peñas, R., Pulham, A., and Taborda, A. 1995. Basin development and tectonic history of the Llanos Basin, Eastern Cordillera, and Middle Magdalena Valley, Colombia. AAPG Bulletin, 79(10): 1421-1443.

Fagin, S. 1996. The fault shadow problem: Its nature and elimination. The Leading Edge, 15(9): 1005-1013.

Fagin, S. 1998 Model-based depth imaging. Society of Exploration Geophysicists. Tulsa, 184p.

Gochioco, L., Novianti, I., and Pascual, R. 2002. Resolving fault shadow problems in Irian Jaya (Indonesia) using prestack depth migration. The Leading Edge, 21(9): 911-920.

Martínez, N., y Agudelo, W. 2009. Efecto de las velocidades en las estructuras subyacentes a fallas sombras de falla. X Simposio Bolivariano Exploración Petrolera en Cuencas Subandinas. Cartagena, Colombia.

Quigley, D., Lau, A., Stewart, K., Yin, C., Mann, A., and Fitzpatrick, A. 2012. Benefits of constraints for velocity modeling a fault shadow: A case study. SEG Annual meeting. Las Vegas, pp. 1-5.

Trinchero, E. 2000. The fault shadow problem as an interpretation pitfall. The Leading Edge, 19(2): 132-135.

Trabajo recibido: noviembre 01 de 2016

Trabajo aceptado: abril 10 de 2017

Manuscrito publicado en internet: abril 06 de 2017 